\title{
An attempt for a unified description of mechanical testing on Zircaloy-4 cladding subjected to simulated LOCA transient
}

\author{
Jean Desquines*, Doris Drouan, Elodie Torres, Séverine Guilbert, and Pauline Lacote \\ PSN-RES/SEREX/LE2M, IRSN, Bâtiment 327, BP 3, 13115 Saint-Paul-Lez-Durance, France
}

Received: 25 September 2015 / Received in final form: 20 January 2016 / Accepted: 28 January 2016

Published online: 25 March 2016

\begin{abstract}
During a Loss Of Coolant Accident (LOCA), an important safety requirement is that the reflooding of the core by the emergency core cooling system should not lead to a complete rupture of the fuel rods. Several types of mechanical tests are usually performed in the industry to determine the degree of cladding embrittlement, such as ring compression tests or four-point bending of rodlets. Many other tests can be found in the open literature. However, there is presently no real intrinsic understanding of the failure conditions in these tests which would allow translation of the results from one kind of mechanical testing to another. The present study is an attempt to provide a unified description of the failure not directly depending on the tested geometry. This effort aims at providing a better understanding of the link between several existing safety criteria relying on very different mechanical testing. To achieve this objective, the failure mechanisms of pre-oxidized and pre-hydrided cladding samples are characterized by comparing the behavior of two different mechanical tests: Axial Tensile (AT) test and "C"-shaped Ring Compression Test (CCT). The failure of samples in both cases can be described by usual linear elastic fracture mechanics theory. Using interrupted mechanical tests, metallographic examinations have evidenced that a set of parallel cracks are nucleated at the inner and outer surface of the samples just before failure, crossing both the oxide layer and the oxygen rich alpha layer. The stress intensity factors for multiple crack geometry are determined for both AT and CCT samples using finite element calculations. After each mechanical test performed on high temperature steam oxidized samples, metallography is then used to individually determine the crack depth and crack spacing. Using these two important parameters and considering the applied load at fracture, the stress intensity factor at failure is derived for each tested sample. This procedure provides an assessment scheme to determine experimentally the fracture toughness of the prior- $\beta$ region in the mid-wall of the oxidized samples. The obtained fracture toughness for CCT and AT samples are thus compared, confirming that the linear elastic fracture mechanics is a relevant tool to describe the strength of LOCA embrittled cladding alloys.
\end{abstract}

\section{Introduction}

Two main types of tests have been developed to check the degree of material embrittlement after high temperature oxidation under steam environment, some combining the influence of applied load and oxidation-induced embrittlement and others relying on the mechanical testing of separately oxidized samples.

A very high degree of prototypicality is obtained using the integral thermal shock test originally developed by JAEA [1-7]. The test sample is a pressurized rodlet with inserted alumina pellets. The sample is inserted in a quartz tube and heated using an infrared lamp furnace. The quartz tube allows injection of steam environment, and at the end

\footnotetext{
* e-mail: jean.desquines@irsn.fr
}

of the test, the rod is quenched by water injection from the bottom end of the quartz tube. An axial tensile (AT) device is also connected to the upper plug of the cladding tube to apply an axial load during the quench. This test reproduces the main phases expected during a LOCA: cladding creep, ballooning and burst, high temperature oxidation under steam environment and finally water quench under applied load. The axial load results from partial restraint of thermal contraction of the rod during the quench under actual LOCA conditions. JAEA performed a large set of such integral thermal shock tests providing a rather simple result at the end of the test: failure or integrity of the rod. The complexity of the phenomena activated during such tests cannot offer a straightforward interpretation of the fuel rod failure conditions for modeling purpose.

In many laboratories, the LOCA consequences on the fuel cladding are rather studied by a sequential testing 
Table 1. Ingot chemical composition of the tested SRA Zry-4.

\begin{tabular}{lllll}
\hline $\begin{array}{l}\mathrm{Sn} \\
(\mathrm{wt} \%)\end{array}$ & $\begin{array}{l}\mathrm{Fe} \\
(\mathrm{wt} \%)\end{array}$ & $\begin{array}{l}\mathrm{Cr} \\
(\mathrm{wt} \%)\end{array}$ & $\begin{array}{l}\mathrm{O} \\
(\mathrm{wt} \%)\end{array}$ & $\begin{array}{l}\mathrm{H} \\
(\mathrm{wppm})\end{array}$ \\
\hline 1.30 & 0.21 & 0.11 & 0.14 & 7 \\
\hline
\end{tabular}

procedure: high temperature steam oxidation of a cladding sample or a fuel rod followed by a low temperature mechanical testing. The steam oxidation is sometimes performed using pressurized rodlets with pellets $[8,9]$ or more simply on cladding samples in many other labs. The mechanical tests are usually: ring compression, AT loading [8], three- or four-point bending $[8,10]$. Such sequential tests can provide results of interest to clarify the failure mode of the rod and expectedly modeling data. However, there is currently no robust procedure to extrapolate the results from one mechanical test to another one.

In the present paper, two different sample geometries, corresponding to extremely different mechanical tests, are subjected to high temperature steam oxidation at $900{ }^{\circ} \mathrm{C}$ followed by mechanical testing at room temperature. The AT test is governed by rather uniform axial stress whereas the "C"-shaped Ring Compression Test (CCT) test is governed by hoop bending load. The failure mode is studied by performing interrupted tests, just before sample failure to better understand the influencing parameters on the oxidized cladding embrittlement. After this, the failure of AT samples is analyzed and compared to the failure conditions of CCT. A unified understanding of the failure conditions of these tests is provided and discussed.

\section{Experiments}

\subsection{Materials}

Stress Relieved Annealed (SRA) low-tin Zry-4 cladding tube with nominal chemical composition described in Table 1 is used in this study. The alloy was manufactured by CEZUS. The outer diameter of the tubes is $9.5 \mathrm{~mm}$ and the cladding thickness $0.57 \mathrm{~mm}$.

\subsection{Testing protocol}

The first step of the testing protocol consists in low temperature oxidation and machining of the test samples. Some of the samples were not oxidized but directly machined. The low temperature $\left(470{ }^{\circ} \mathrm{C}\right)$ oxidation under wet-air environment induces several hundred wppm hydrogen charging in the samples and a thin zirconia layer is formed at the inner and outer diameter of the sample.

Two sample geometries were considered in this preliminary step of the testing protocol:

- $20 \mathrm{~mm}$ long ring samples;

- AT samples with geometry specified in Figure 1.
The ring samples were obtained by diamond saw cutting whereas the AT samples were spark machined in order to avoid residual stress deposition in the gage sections.

After this step, the samples were high temperature oxidized, at both inner and outer surfaces, in a vertical furnace heated at $900{ }^{\circ} \mathrm{C}$ under steam environment. This temperature is relevant for small-break LOCA studies. The water quench was simulated by dropping the sample at $900{ }^{\circ} \mathrm{C}$ into a water bath. The steam oxidation protocol and its qualification are detailed in references [11-13]. The samples were weighted before and after $900{ }^{\circ} \mathrm{C}$ steam oxidation to determine a measured Equivalent Cladding Reacted (ECR). The ECR is a key parameter influencing the material embrittlement. It is defined as the ratio between the weight gain versus its maximum possible value corresponding to full oxidation of the sample.

The $20 \mathrm{~mm}$ long ring samples were then divided into two 10-mm long rings and one edge of the ring samples was then cut, using a diamond wire saw, to form "C"-shaped samples.

After the high temperature oxidation, the AT samples and CCT samples were subjected to mechanical testing, using an INSTRON 5566 electromechanical test device, to determine the degree of material embrittlement. The CCT test consists in compressing the sample as illustrated in Figure 2 at a displacement rate of $1 \mathrm{~mm} / \mathrm{min}$ (see Refs. $[14,15]$ for complementary details). After mechanical testing, upper and lower parts of AT samples were reused to machine $10 \mathrm{~mm}$ long "C"-shaped ring compression samples. Post-test analyses were additionally performed to clarify the material failure mode, such as: metallography and hydrogen content measurements. Hydrogen contents are measured by sample fusion using a Brücker ONH mat 286 device.

\subsection{Test matrixes}

As explained above, some of the samples were hydrogen charged using wet-air oxidation at $470{ }^{\circ} \mathrm{C}$. A thin oxidation layer was also formed during this period. This protocol leads to about $10 \%$ uncertainty on hydrogen content and about one micrometer variation in oxide layer thickness.

Three main test matrixes were defined, dedicated to the mechanical behavior of $900{ }^{\circ} \mathrm{C}$ steam oxidized cladding samples:

- test matrix \#1: two pairs of CCT tests on steam oxidized samples, each pair corresponds to the samples originating from the same $20 \mathrm{~mm}$ long steam oxidized ring, one is loaded to failure and the second is interrupted just before failure load;

- test matrix \#2: six CCT tests on steam oxidized cladding samples with hydrogen content below 1000 wppm;

- test matrix \#3: eight AT samples, steam oxidized, were tested with hydrogen contents ranging between asreceived content up to about 1000 wppm.

The first test matrix aims at clarifying the failure mode of oxidized cladding samples. The second and the third test matrixes provide respectively determination of the failure behavior of CCT samples and AT samples for comparison. 

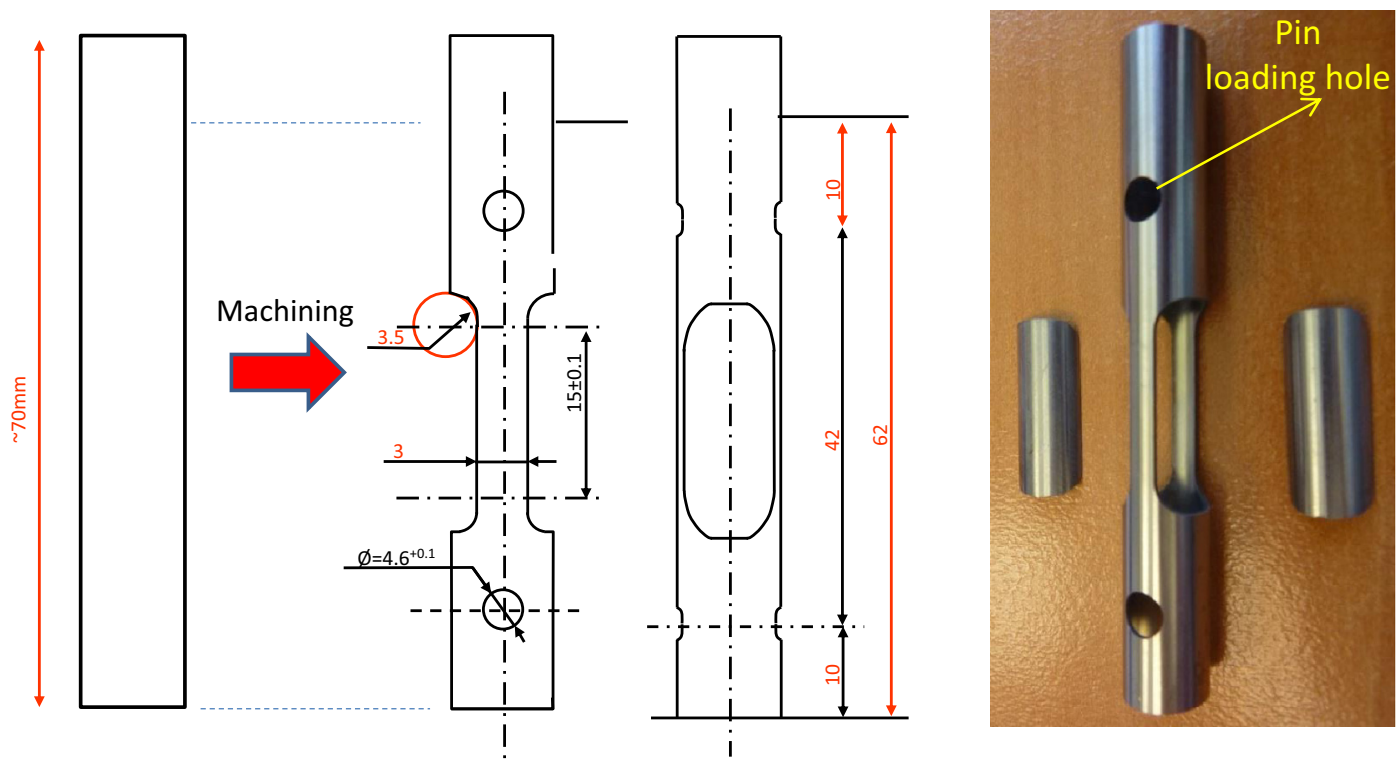

Fig. 1. Axial tensile sample geometry.
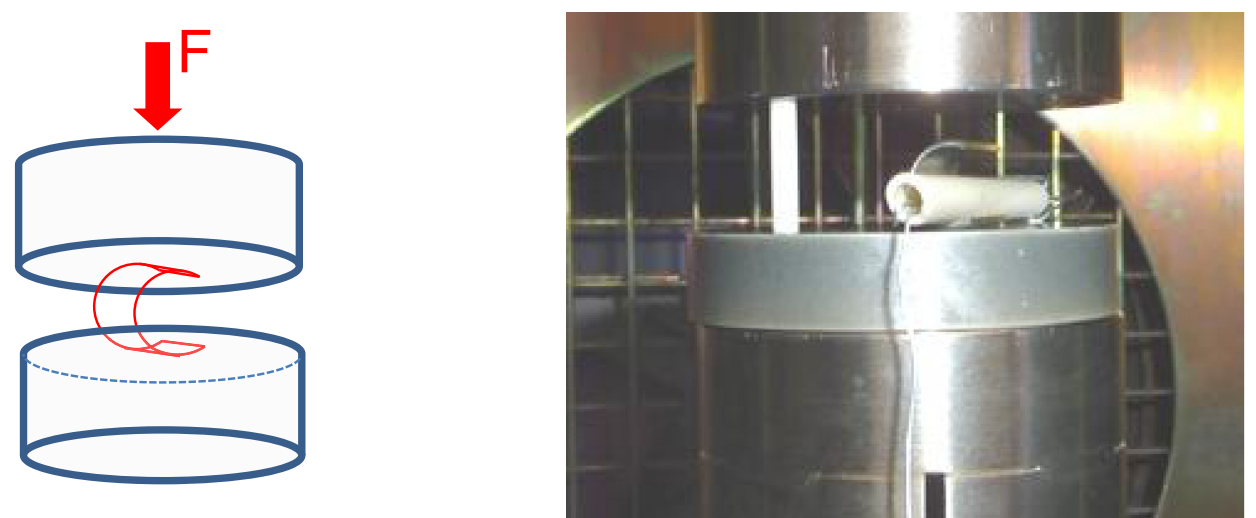

Fig. 2. Principle of "C"'shaped ring Compression Test (CCT) between two flat surfaces.

\section{Test results}

\subsection{Oxidation results}

The main oxidation results associated to each mechanical test are summarized in Table 2. The oxide layer thickness and the oxygen stabilized $\alpha(\mathrm{O})$ layer thickness are measured on post-test metallography after both low temperature oxidation and steam oxidation. A key parameter influencing material embrittlement is the sample hydrogen content $([\mathrm{H}])$, the measured value is provided with two standard deviations uncertainty after high temperature steam oxidation. The protective effect of pre-existing oxide layer explains some very low ECR values and sometimes zero weight gain values.

\subsection{Mechanical test results}

The key mechanical test results are summarized in Table 3. A failure mode is determined for each sample: ductile or brittle. A ductile failure is associated to samples developing macroscopic plastic deformation or stable crack growth prior to failure, all others are considered as brittle.

The CCT test load is normalized by sample axial length $(L)$. During CCT testing, the hoop bending stress is heterogeneous and its maximum value is obtained at outer diameter and equatorial location. This maximum hoop stress value is determined from the normalized applied load in the elastic range according to references $[14,15]$ :

$$
\sigma_{\max }(\mathrm{MPa})=\frac{1}{1.2987 \times 10^{-2}} \frac{F}{L}(\mathrm{~N} / \mathrm{mm}) .
$$

The CCT sample failure is expected to be governed by this stress value in the brittle range.

For AT testing, the axial stress $\left(\sigma_{\max }\right.$ in Tab. 3$)$ is assumed to be homogeneous and can be determined straightforward as the ratio between applied load and gage cross-section. However, it is clear that there are material properties heterogeneities in the various layers of the oxidized samples. But it is reasonable to consider that the equivalent homogeneous material properties are acceptably 
Table 2. Low temperature oxidation conditions and $900{ }^{\circ} \mathrm{C}$ oxidation results for the test matrix.

\begin{tabular}{|c|c|c|c|c|c|c|c|c|}
\hline \multirow[b]{2}{*}{$\begin{array}{l}\text { Test } \\
\text { Matrix } \\
(\#)\end{array}$} & \multirow[b]{2}{*}{$\begin{array}{l}\text { Sample } \\
(\#)\end{array}$} & \multicolumn{2}{|c|}{$470^{\circ} \mathrm{C}$ wet-air oxidation } & \multicolumn{5}{|c|}{$900^{\circ} \mathrm{C}$ steam oxydation } \\
\hline & & $\begin{array}{l}\text { Time } \\
\text { (days) }\end{array}$ & $\begin{array}{l}\mathrm{ZrO}_{2} \text { layer } \\
\text { thickness } \\
\pm 1 \mu \mathrm{m} \\
(\mu \mathrm{m})\end{array}$ & $\begin{array}{l}\text { Time } \\
\text { (min) }\end{array}$ & $\begin{array}{l}\mathrm{ZrO}_{2} \text { layer } \\
\text { thickness } \\
\pm 1 \mu \mathrm{m} \\
(\mu \mathrm{m})\end{array}$ & $\begin{array}{l}\alpha(\mathrm{O}) \text { layer } \\
\text { thickness } \\
\pm 1 \mu \mathrm{m} \\
(\mu \mathrm{m})\end{array}$ & (wppm) & $\begin{array}{l}\text { Measured } \\
\text { ECR } \\
(\%)\end{array}$ \\
\hline \multirow[t]{4}{*}{1} & CCT1 & 15 & 8 & 15 & 8 & 28 & $430 \pm 50$ & 1.2 \\
\hline & $\mathrm{CCT} 2$ & 15 & 8 & 30 & 10 & 22 & $590 \pm 60$ & 3.2 \\
\hline & CCT3 & 15 & 8 & 15 & 8 & 28 & $430 \pm 40$ & 1.2 \\
\hline & $\mathrm{CCT} 4$ & 15 & 8 & 30 & 10 & 30 & $610 \pm 80$ & 3.2 \\
\hline \multirow[t]{6}{*}{2} & $\mathrm{CCT} 5$ & 15 & 7.5 & 5 & 7.5 & 19 & $370 \pm 50$ & 0.0 \\
\hline & CCT6 & 15 & 7.5 & 15 & 9.5 & 31 & $630 \pm 100$ & 1.2 \\
\hline & $\mathrm{CCT} 7$ & 15 & 6.5 & 30 & 9.5 & 36 & $700 \pm 90$ & 3.4 \\
\hline & $\mathrm{CCT} 8$ & 20 & 9.5 & 60 & 22 & 26 & $970 \pm 110$ & 5.5 \\
\hline & ССТ9 & 20 & 9.5 & 60 & 22 & 27 & $1060 \pm 110$ & 5.5 \\
\hline & CCT10 & 20 & 9.5 & 60 & 24.5 & 27 & $1030 \pm 100$ & 5.5 \\
\hline \multirow[t]{8}{*}{3} & AT1 & 20 & 9.5 & 60 & 22 & 30 & $970 \pm 120$ & 5.5 \\
\hline & AT2 & 20 & 9.5 & & & & $1060 \pm 110$ & 5.5 \\
\hline & AT3 & 20 & 10 & 60 & 27.5 & 26 & $1070 \pm 120$ & 5.5 \\
\hline & AT4 & 15 & 8 & 5 & 7.5 & 12 & $370 \pm 50$ & 0.0 \\
\hline & AT5 & 15 & 8 & 15 & 9.5 & 19 & $630 \pm 100$ & 1.2 \\
\hline & AT6 & 15 & 8 & 30 & 9.5 & 36 & $700 \pm 160$ & 3.4 \\
\hline & AT7 & 0 & 0 & 15 & 13 & 21 & $40 \pm 20$ & 3.8 \\
\hline & AT8 & 0 & 0 & 30 & 16.5 & 20 & $40 \pm 10$ & 4.8 \\
\hline
\end{tabular}

represented by the most resistant phase property which is not cracked and is consequently affected by the strongest stress levels. This is a common assumption for materials affected by a cracked brittle surface layer. The failure axial load is extrapolated to the expected value for an entire fuel cladding $(\mathrm{F} / \mathrm{rod})$ without any machined gage section.

Before mechanical testing, some incipient cracks were observed in the oxygen stabilized $\alpha(\mathrm{O})$ layer. Similar cracks were observed by Nagase and Fuketa [4] and Kim et al. [16] and most probably form during the water quench. When comparing CCT3 interrupted test (Fig. 3) and CCT1 twin sample loaded up to failure (Fig. 4) many nucleated cracks propagating through the zirconia layer and the oxygen stabilized $\alpha(\mathrm{O})$ layer were observed along the sample outer surface. The observed cracks never propagated into $\alpha(\mathrm{O})$ inclusions in the $\alpha+$ prior- $\beta$ region of the cladding sample. These two samples confirm that the brittle failure of CCT samples is governed by crack instability. The relevant parameter to describe the intensity of the stress singularity at the crack tip is the stress intensity factor $\left(K_{I}\right)$. The critical value of this parameter under plane strain loading is the sample fracture toughness $\left(K_{I c}\right)$. Similar conclusions were obtained comparing CCT2 and CCT4 interrupted test on twin material. The procedure to determine the stress intensity factor is described in the following.

It is well known that multiple crack nucleation has a shadowing effect $[2,17]$ limiting the stress intensity factor at the crack tips. It was thus decided to measure the crack spacing on metallographic samples. For CCT tests, the crack spacing was considered to be the largest average value (left and right) associated to each observed crack. The measured crack spacing for each CCT sample is reported in Table 3 . It is normally considered under uniform applied stress, that the largest stress intensity factor is obtained on the edge of multiple nucleated crack set. Considering CCT sample, the situation is different because the applied stress (assuming no crack nucleated) at the sample surface decreases when moving away from equator. For this reason, the maximum stress intensity factor is considered to be obtained within the nucleated crack set.

Considering now, the AT test with uniform applied stress, the worst location associated with maximum stress intensity factor corresponds to the edge of the multiple nucleated cracks set. The measured crack spacing for AT tests is also reported in Table 3 .

The mechanical test results are analyzed in the next paragraph after calculation of the stress intensity factor associated to each sample geometry. The Cast3m finite element code ${ }^{1}$ developed by CEA was used for the calculations.

\footnotetext{
$\overline{{ }^{1} \text { http://www-cast3m.cea.fr/ }}$
} 
Table 3. Mechanical test results and determination of the sample fracture toughness.

\begin{tabular}{|c|c|c|c|c|c|c|c|c|c|}
\hline \multirow[b]{2}{*}{ Test Matrix } & \multirow[b]{2}{*}{ Sample } & \multicolumn{2}{|c|}{ Failure } & \multirow{2}{*}{$\begin{array}{l}F / L \\
(\mathrm{~N} / \mathrm{mm})\end{array}$} & \multirow{2}{*}{$\begin{array}{l}\mathrm{F} / \mathrm{rod} \\
(\mathrm{N})\end{array}$} & \multirow{2}{*}{$\begin{array}{l}\sigma_{\max } \\
(\mathrm{MPa})\end{array}$} & \multirow{2}{*}{$\begin{array}{l}\lambda: \text { crack spacing } \\
( \pm 1 \mu \mathrm{m}) \\
(\mu \mathrm{m})\end{array}$} & \multirow{2}{*}{$\begin{array}{l}a: \text { crack depth } \\
( \pm 1 \mu \mathrm{m}) \\
(\mu \mathrm{m})\end{array}$} & \multirow{2}{*}{$\begin{array}{l}K_{I c} \\
(\mathrm{MPa} \sqrt{ } \mathrm{m})\end{array}$} \\
\hline & & $(\mathrm{y} / \mathrm{n})$ & Mode & & & & & & \\
\hline \multirow[t]{4}{*}{1} & $\mathrm{CCT} 1$ & $\mathrm{y}$ & Brittle & 15.5 & & 1190 & 50 & 36 & 7.2 \\
\hline & CCT2 & $\mathrm{y}$ & Brittle & 11.2 & & 865 & 85 & 32 & 6.3 \\
\hline & CCT3 & $\mathrm{n}$ & & 14.2 & & 1090 & 50 & 36 & $6.6>$ \\
\hline & $\mathrm{CCT} 4$ & $\mathrm{n}$ & & 9.9 & & 765 & 185 & 40 & $7.4>$ \\
\hline \multirow[t]{6}{*}{2} & CCT5 & $\mathrm{y}$ & Ductile & 63.8 & & 4910 & 30 & 27 & \\
\hline & CCT6 & $\mathrm{y}$ & Brittle & 17.3 & & 135 & 62 & 40 & 8.7 \\
\hline & $\mathrm{CCT7}$ & $\mathrm{y}$ & Brittle & 17.0 & & 1310 & 100 & 45 & 10.3 \\
\hline & $\mathrm{CCT} 8$ & $\mathrm{y}$ & Brittle & 9.8 & & 755 & 80 & 48 & 5.4 \\
\hline & ССТ9 & $\mathrm{y}$ & Brittle & 11.1 & & 855 & 90 & 51 & 6.5 \\
\hline & СCT10 & $\mathrm{y}$ & Brittle & 9.1 & & 700 & 56 & 51 & 4.2 \\
\hline \multirow[t]{8}{*}{3} & AT1 & $\mathrm{y}$ & Brittle & & 4860 & 295 & $>1000$ & 52 & 4.2 \\
\hline & $\mathrm{AT} 2^{\mathrm{a}}$ & $\mathrm{y}$ & Brittle & & & & & & \\
\hline & AT3 & $\mathrm{y}$ & Brittle & & 4890 & 295 & 800 & 54 & 4.2 \\
\hline & AT4 & $\mathrm{y}$ & Ductile & & 13790 & 855 & 49 & 20 & \\
\hline & AT5 & $\mathrm{y}$ & Brittle & & 9660 & 600 & 65 & 28 & 5.2 \\
\hline & AT6 & $\mathrm{y}$ & Brittle & & 7600 & 470 & $>1000$ & 45 & 5.0 \\
\hline & $\mathrm{AT}^{\mathrm{b}}$ & $\mathrm{y}$ & Ductile & & 11450 & 705 & 18 & 21 & \\
\hline & $\mathrm{AT}^{\mathrm{b}}$ & $\mathrm{y}$ & Ductile & & 11930 & 730 & 20 & 24 & \\
\hline
\end{tabular}

${ }^{a}$ Sample failure when mounting.

${ }^{\mathrm{b}}$ Oxide layer spalling during the mechanical test.

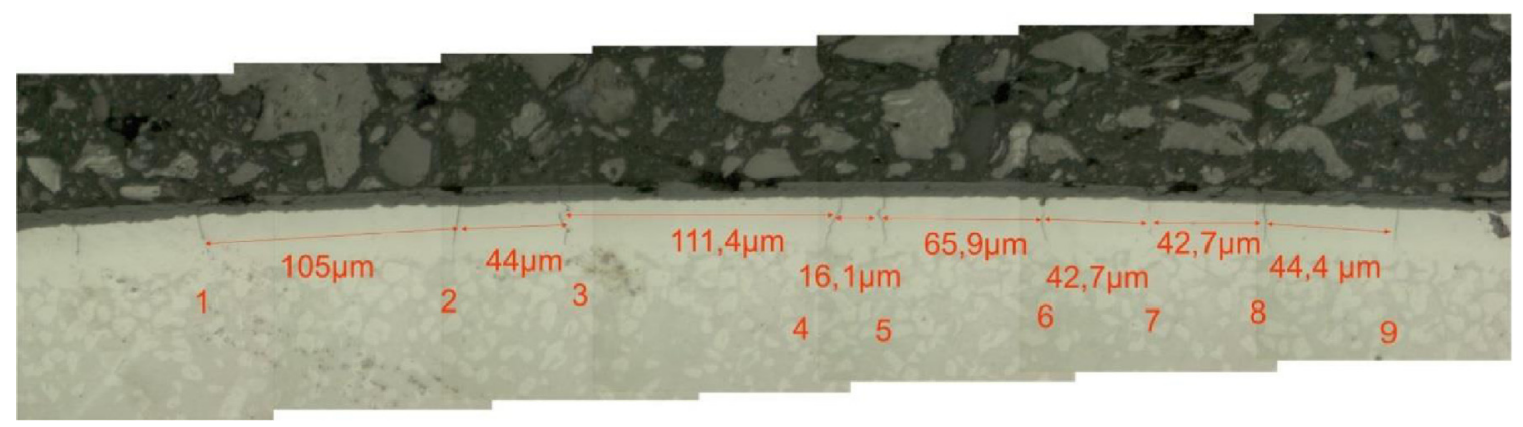

Fig. 3. Crack nucleation and crack spacing at outer diameter equatorial location of CCT3 interrupted CCT test at $92 \%$ of the failure load (comparison to CCT1 test twining sample).

\section{Analysis of the mechanical test results}

\subsection{Modeling CCT test with multiple crack nucleation at outer diameter}

A two-dimensional finite element model describing the elastic behavior of a CCT sample with a set of regularly spaced cracks nucleated at its outer surface was developed. The nominal geometry of the cladding is $9.5 \mathrm{~mm}$ outer diameter and $0.57 \mathrm{~mm}$ cladding thickness. The crack spacing and crack depth are parameters of this model. The contact conditions between crack lips are incorporated as boundary conditions.
The shape function at equatorial location, $\Phi\left(\frac{\lambda}{e}, \frac{a}{e}\right)$, describing the geometry influence on the stress intensity factor, was tabulated for various crack set geometries and the consistency with single crack nucleated $\left(\frac{\lambda}{e}=+\infty\right)$ was checked. A correlation is established providing the $\Phi$ value for all possible crack geometries (see Fig. 5). This shape function was used to determine the stress intensity factor value for CCT tests in Table 3. Figure 5 confirms that the stress intensity factor increases with increasing crack spacing. This phenomenon is known as shadowing effect of neighbor parallel cracks. A sensitivity study that is not reported in the present paper showed that the stress intensity factor decreases considering cracks away from equatorial location. 


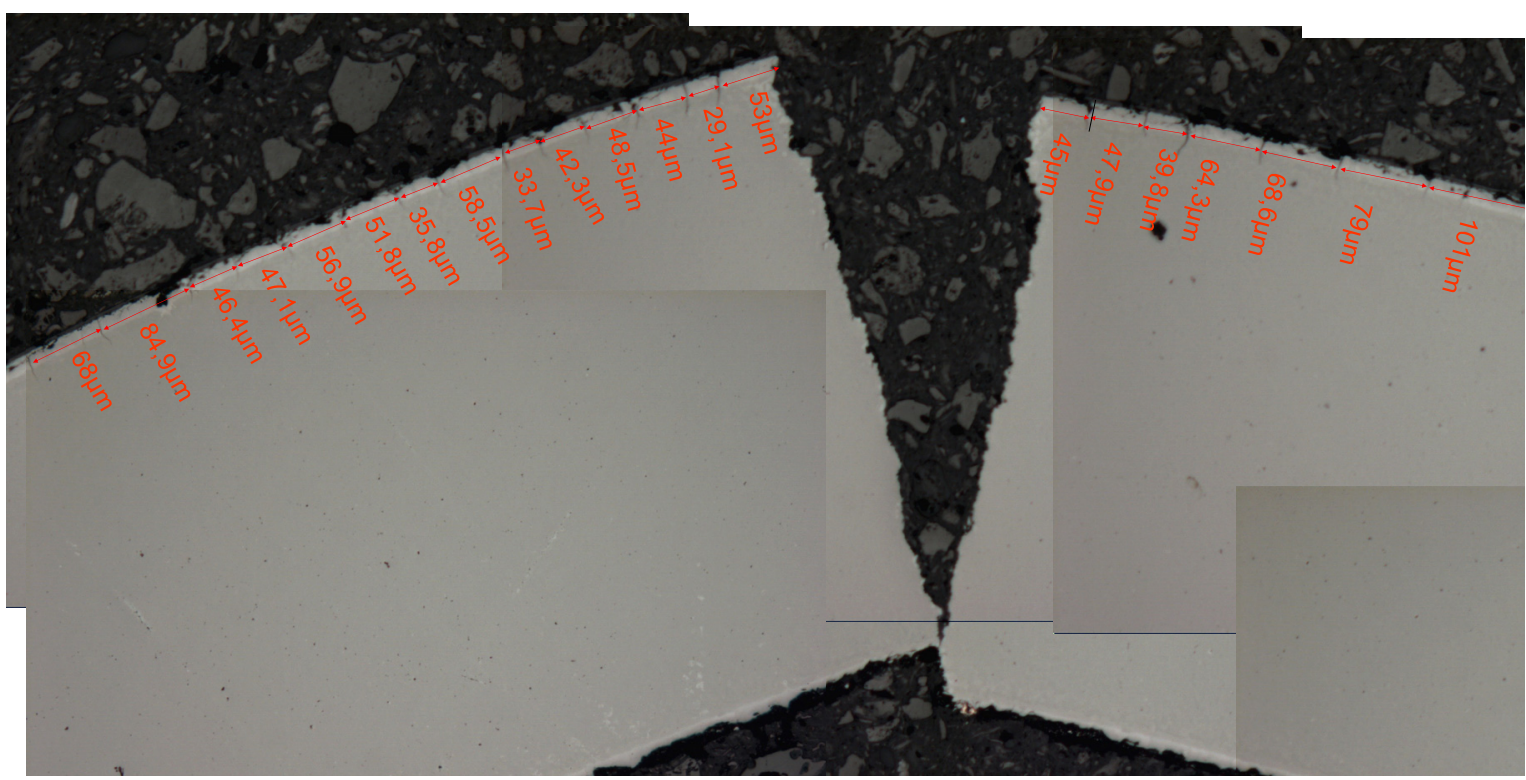

Fig. 4. Crack nucleation and crack spacing at outer diameter equatorial location of CCT1 after failure.

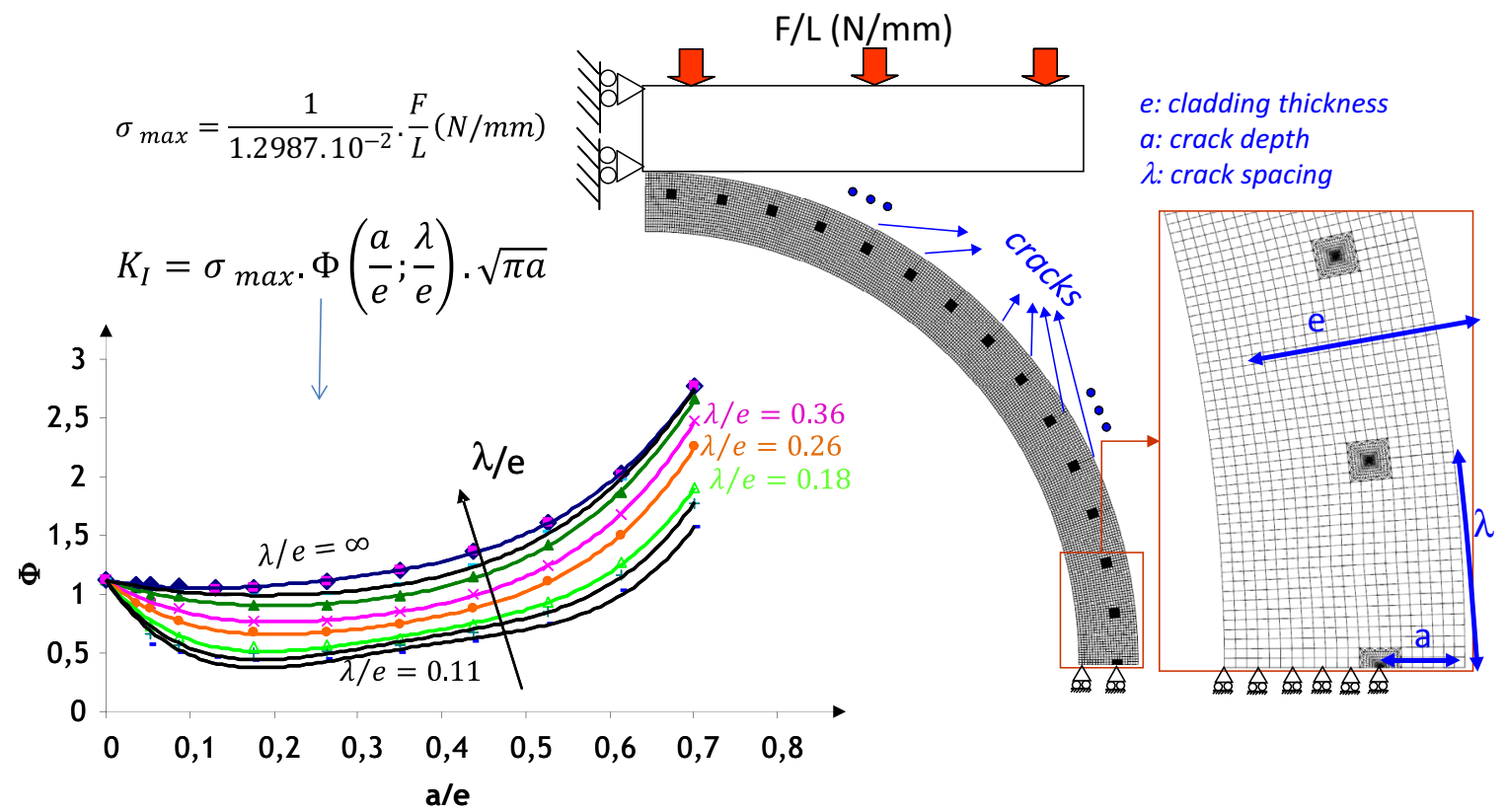

Fig. 5. Stress intensity factor at outer diameter equatorial location during CCT with multiple crack nucleation (marks: calculated values; lines: correlated values).

\subsection{Modeling axial tensile tests with multiple crack nucleation at outer diameter}

Using a similar approach, the multiple crack nucleation along the legs of the AT sample was studied using finite element simulations. The largest value of the stress intensity factor (see Fig. 6) is obtained at the edge of the array of parallel cracks. Consequently, the sample failure is expected at this location. In order to describe accurately multiple crack influence, a set of nine regularly spaced cracks subjected to tension was modeled using a 2D finite element model considering the sample symmetries, as illustrated in Figure 6. Murakami [2] shows that the crack number influence is close to saturated above about five cracks. There is consequently no need for additional cracks. The shadowing effect also disappears at large crack spacing, this can be easily described using the nine-crack model. The crack number is considered as sufficiently large to have no influence on the stress intensity factor of the edge crack. For crack clusters with varying crack spacing, the stress intensity factor at the edge is rather governed by the spacing between the two edge cracks. The crack depth and crack spacing are the key parameters of the modeling. 

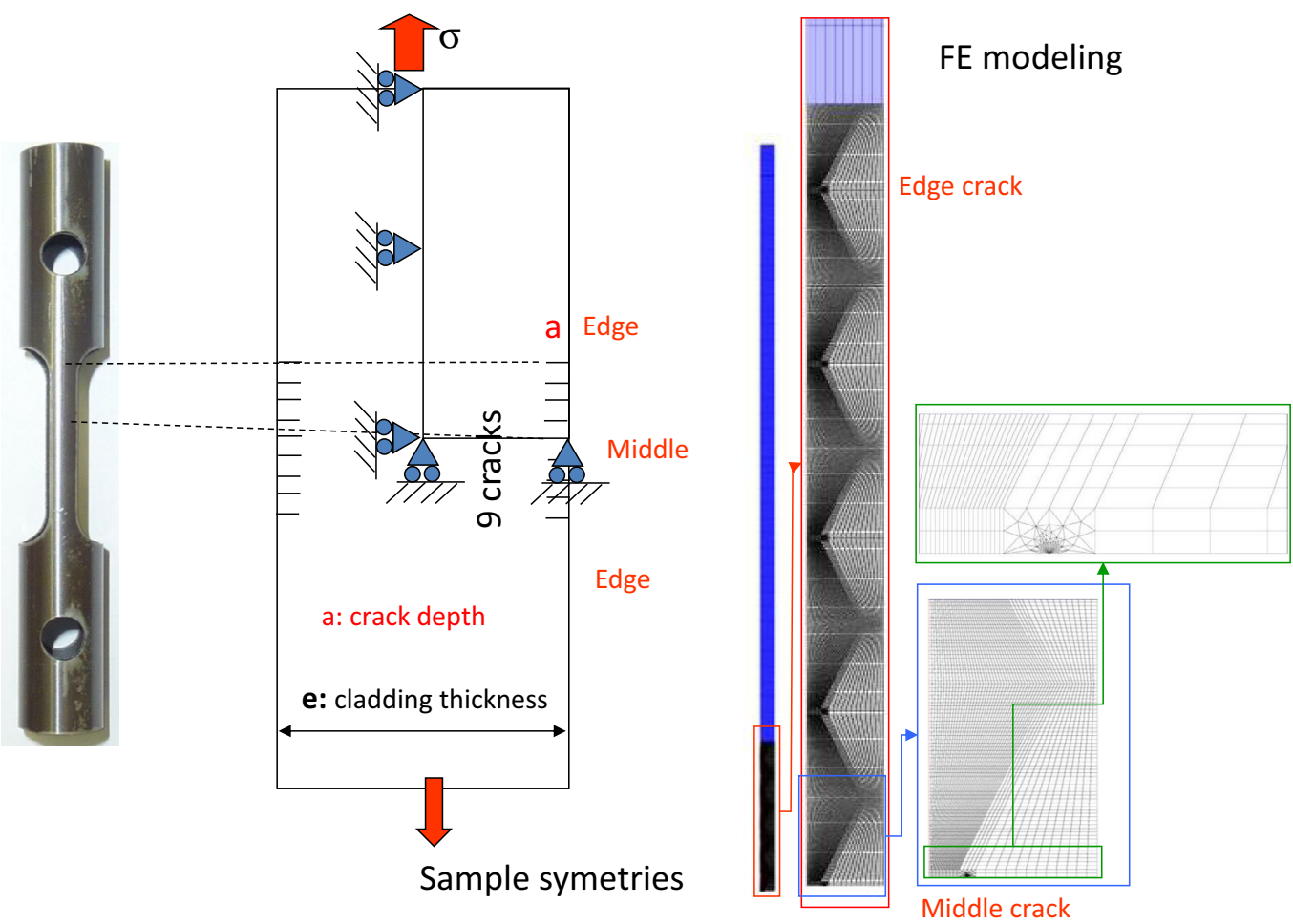

Fig. 6. Periodic crack array modeling to simulate an axial tensile test with multiple crack nucleation at its inner and outer surface.
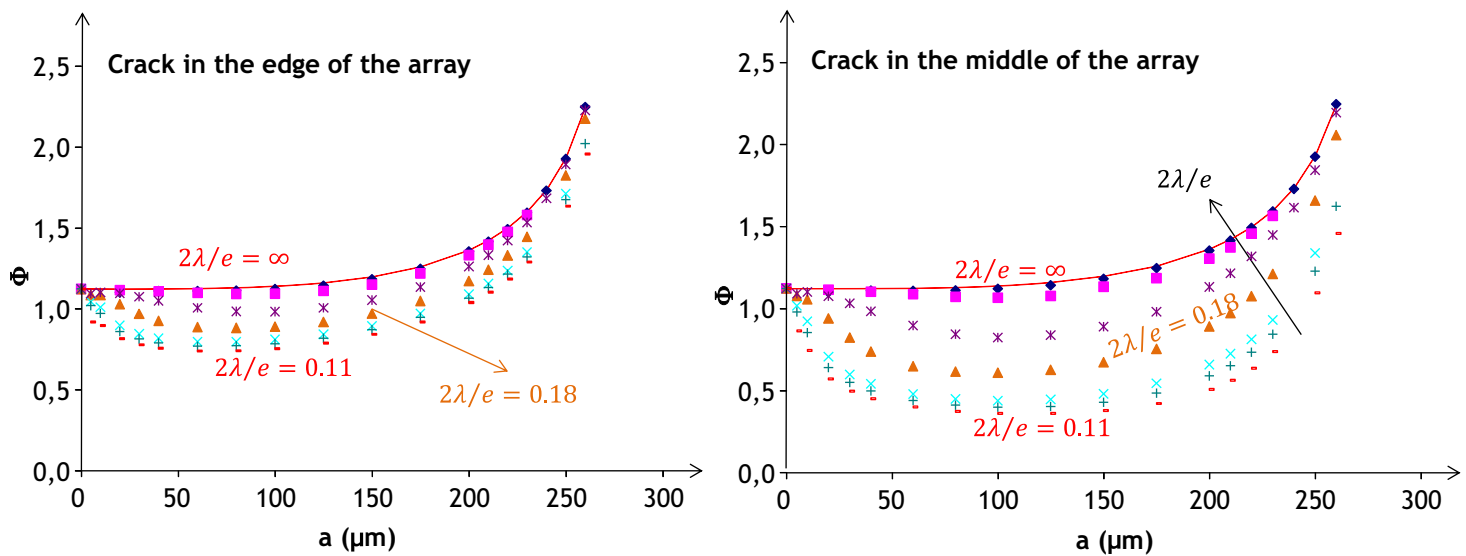

Fig. 7. Shape function values at the edge and at the middle location of the crack array (marks: calculated values; lines: correlated values).

The stress intensity factor is determined as previously done for CCT by incorporating a shape function describing the combined influence of normalized crack depth and crack spacing: $K_{I}=\sigma \Phi\left(\frac{2 a}{e} ; \frac{2 \lambda}{e}\right) \sqrt{\pi a}$.

The shape function was tabulated at edge crack and middle crack as illustrated in Figure 7. The values at both locations are comparable for large crack spacing and significantly higher at the edge of the array, as expected, for close cracks. In other words, when a dense array of cracks is nucleated, the fracture is expected at the edge of the AT sample gage section. On the contrary, when only few cracks with large crack spacing are nucleated, the stress intensity is expected to be comparable at any crack tip of the array, the failure is expected anywhere in the gage section. The edge crack shape function was used to determine the stress intensity factors values reported in Table 3 for AT tests.

\subsection{Evaluation of CCT and AT fracture toughness values}

The fracture toughness values are valid only for brittle samples, other parameters are required to describe the ductile failures. The obtained values using the abovedescribed procedure are reported in Figure 8. The values are rather low considering the usual values obtained for metals and are close to expected range for ceramics, especially at large hydrogen content. 


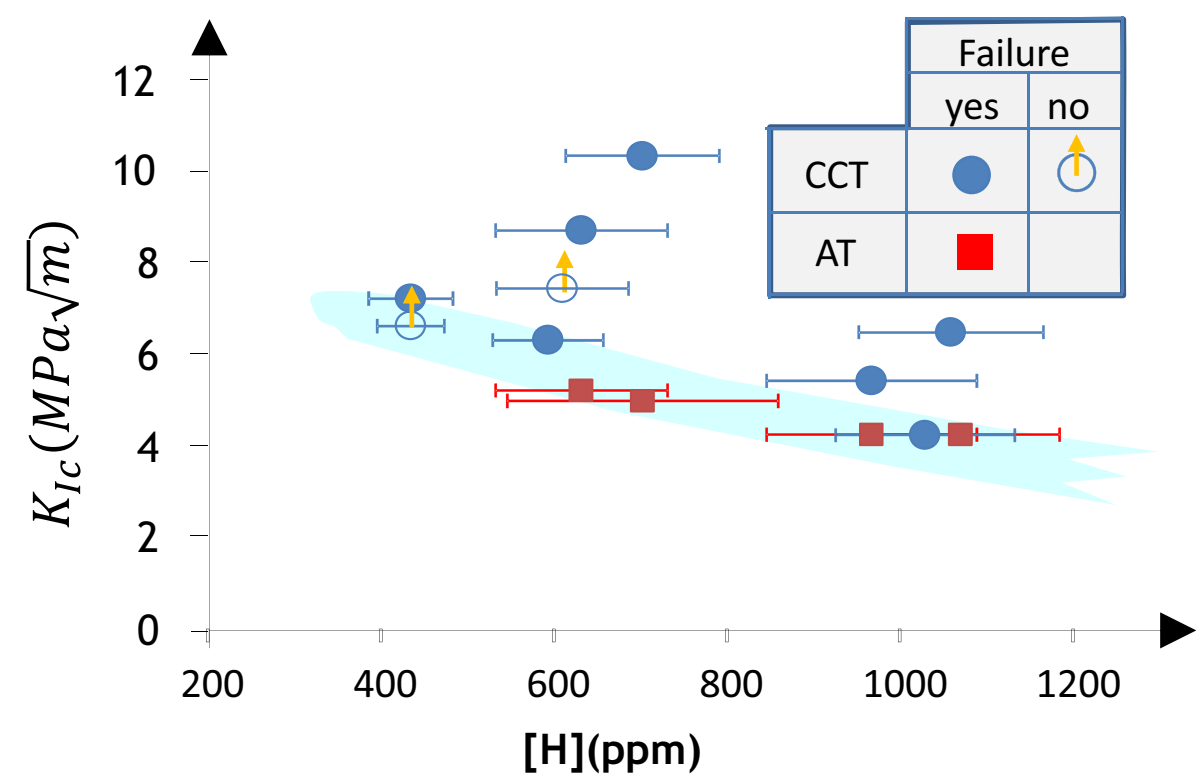

Fig. 8. Influence of sample hydrogen content on the evaluated cladding fracture toughness.

The fracture toughness of the AT tests corresponds to the lower trend of CCT tests. This might be linked to the limited volume of material exposed to the maximum stress value during a CCT compared to the gage volume of an $\mathrm{AT}$ test. If the crack instability happens slightly away from equatorial location, the stress intensity is clearly overestimated using the value at equator. However, there is an acceptable consistency between the obtained fracture toughness values after $900^{\circ} \mathrm{C}$ steam oxidation. An average value representing the average trend of $\mathrm{AT}$ tests and the lower trend of CCT tests is plotted in blue in Figure 8, this curve represents the expected value of material fracture toughness for large scale samples.

\section{Conclusion}

The room temperature mechanical behavior of cladding samples exposed to high temperature steam oxidation has been analyzed in the present paper. The mechanical behavior of the brittle samples appears consistent with linear elastic fracture mechanics. The cracks responsible for material embrittlement are nucleated in the oxygen stabilized $\alpha(\mathrm{O})$ layer during the sample quench and some of them propagate through the oxide layer during the mechanical test. A set of parallel crack forms at the oxidized sample surface. The sample failure was obtained for $K_{I c}$ (critical stress intensity factor or material fracture toughness) that is comparable during CCT and AT tests. However, the fracture toughness was slightly lower during AT tests. This was attributed to scale effect associated to very different gage volumes exposed to the maximum stress in the two kinds of tests. A large number of CCT tests would then be required to determine the lower fracture toughness expected on large scale samples.

The crack spacing is an influencing parameter for brittle samples with significant strength (close to the ductilebrittle transition), but for samples subjected to strong embrittlement a single crack nucleation is expected before sample failure.

The fracture toughness appears as an interesting parameter to describe the post-quench cladding embrittlement after a LOCA. The quantitative comparison between AT and CCT test results is possible using this material parameter.

To the authors knowledge, linear elastic fracture mechanics was surprisingly never evaluated to describe post-LOCA cladding embrittlement and appears as a powerful approach to model the cladding failure. Further extrapolation at higher temperature $\left(1200^{\circ} \mathrm{C}\right)$ and for a large set of ECR is now required for full validation of the proposed methodology. Some difficulties are expected when transposing this approach to Nb-bearing alloys for which the boundary between the oxygen stabilized $\alpha$-layer and the prior $\beta$-layer is not well defined.

\section{References}

1. K. Honma, S. Doi, M. Ozawa, S. Urata, T. Sato, Thermalshock behavior of PWR high burnup fuel cladding under simulated LOCA conditions, in ANS 2001 Annual Meeting, Milwaukee, Wisconsin, USA, June 17-21 (2001)

2. Y. Murakami, Stress intensity factors handbook (Pergamon Press, 1987), Vols. 1, 2

3. F. Nagase, T. Fuketa, Effect of pre-hydriding on thermalshock resistance of Zircaloy-4 cladding under simulated lossof-coolant conditions, J. Nucl. Sci. Technol. 41, 723 (2004)

4. F. Nagase, T. Fuketa, Behavior of pre-hydrided Zircaloy-4 cladding under simulated LOCA conditions, J. Nucl. Sci. Technol. 42, 209 (2005)

5. F. Nagase, Fracture behavior of irradiated Zircaloy-4 cladding under simulated LOCA conditions, J. Nucl. Sci. Technol. 43, 1114 (2006)

6. F. Nagase, T. Chuto, T. Fuketa, Behavior of high burnup fuel cladding under LOCA conditions, J. Nucl. Sci. Technol. 46, $763(2009)$ 
7. F. Nagase, Status and plan of LOCA studies at JAEA, in Fuel Safety Research Meeting, May 19-20, 2010, Tokai, Japan (2010)

8. M.C. Billone, Assessment of current test methods for postLOCA cladding behavior, NUREG Report, CR-7139, 2012

9. J. Stuckert, M. Grosse, C. Rössger, M. Klimenkov, M. Steinbrück, M. Walter, QUENCH-LOCA program at KIT on secondary hydriding and results of the commissioning bundle test QUENCH-L0, Nucl. Eng. Des. 255, 185 (2013)

10. J.-C. Brachet, J. Pelchat, D. Hamon, R. Maury, P. Jacques, J.-P. Mardon, Mechanical behavior at room temperature and metallurgical study of low-tin Zy-4 and M5TM (Zr-NbO) alloys after oxidation at $1100{ }^{\circ} \mathrm{C}$ and quenching, in Proceedings of TCM on Fuel behavior under transient and LOCA conditions, Organized by IAEA, Halden, September 10-14, 2001 (2001)

11. C. Duriez, S. Guilbert, A. Stern, C. Grandjean, L. Belovsky, J. Desquines, Characterization of oxygen distribution in LOCA situations, J. ASTM Int. 8 (2011). DOI:10.1520/JAI103156

12. S. Guilbert, C. Duriez, C. Grandjean, Influence of a pre-oxide layer on oxygen diffusion and on post-quench mechanical properties of Zircaloy- 4 after steam oxidation at $900{ }^{\circ} \mathrm{C}$, in Proceedings of 2010 LWR Fuel Performance/TopFuel/
WRFPM, Orlando, Florida, USA, September 26-29, 2010 (2010), Paper 121

13. S. Guilbert, P. Lacote, G. Montigny, C. Duriez, J. Desquines, C. Grandjean, Effect of Pre-oxide on Zircaloy-4 hightemperature steam oxidation and post-quench mechanical properties, ASTM STP 1523 (2013)

14. J. Desquines, D. Drouan, P. March, S. Fourgeaud, C. Getrey, V. Elbaz, M. Philippe, Characterization of radial hydride precipitation in Zy-4 using "C"-Shaped samples, in LWR Fuel Performance Meeting Topfuel 2013, Charlotte, North Carolina (2013)

15. J. Desquines, D. Drouan, M. Billone, M.P. Puls, P. March, S. Fourgeaud, C. Getrey, V. Elbaz, M. Philippe, Influence of temperature and hydrogen content on stress-induced radial hydride precipitation in Zircaloy-4 cladding, J. Nucl. Mater. 453, 131 (2014)

16. J.H. Kim, B.K. Choi, J.H. Baek, Y.H. Jeong, Effects of oxide and hydrogen on the behavior of Zircaloy-4 cladding during the loss of the coolant accident (LOCA), Nucl. Eng. Des. 236, 2383 (2006)

17. Z.D. Jiang, A. Zeghloul, G. Bezine, J. Petit, Stress intensity factors of parallel cracks in a finite width sheet, Eng. Fract. Mech. 35, 1073 (1990)

Cite this article as: Jean Desquines, Doris Drouan, Elodie Torres, Séverine Guilbert, Pauline Lacote, An attempt for a unified description of mechanical testing on Zircaloy-4 cladding subjected to simulated LOCA transient, EPJ Nuclear Sci. Technol. 2, 13. (2016) 